\title{
Prospective pan-cancer germline testing using MSK-IMPACT informs clinical translation in 751 patients with pediatric solid tumors
}

\author{
Elise M. Fiala ${ }^{1,2,7}$, Gowtham Jayakumaran ${ }^{3,7}$, Audrey Mauguen ${ }^{4,7}$, Jennifer A. Kennedy ${ }^{1,2}$, \\ Nancy Bouvier', Yelena Kemel ${ }^{15}{ }^{5}$, Megan Harlan Fleischut ${ }^{2}$, Anna Maio ${ }^{2}$, Erin E. Salo-Mullen ${ }^{2}$, \\ Margaret Sheehan², Angela G. Arnold², Alicia Latham², Maria I. Carlo ${ }^{2}{ }^{2}$, Karen Cadoo², \\ Semanti Murkherjee², Emily K. Slotkin', Tanya Trippett', Julia Glade Bender ${ }^{1}$, Paul A. Meyers ${ }^{1}{ }^{1}$, \\ Leonard Wexler', Filemon S. Dela Cruz', Nai-Kong Cheung ${ }^{\circledR 1}$, Ellen Basu', Alex Kentsis', Michael Ortiz', \\ Jasmine H. Francis' ${ }^{6}$, Ira J. Dunkel', Yasmin Khakoo', Stephen Gilheeney', Sameer Farouk Sait', \\ Christopher J. Forlenza', Maria Sulis', Matthias Karajannis ${ }^{1}{ }^{1}$, Shakeel Modak', Justin T. Gerstle ${ }^{6}$, \\ Todd E. Heaton ${ }^{6}$, Stephen Roberts' ${ }^{1}$ Ciyu Yang ${ }^{3}$, Sowmya Jairam ${ }^{3}$, Joseph Vijai ${ }^{2}{ }^{2}$, Sabine Topka ${ }^{5}$, \\ Danielle N. Friedman', Zsofia K. Stadler ${ }^{2}$, Mark Robson ${ }^{1}{ }^{2}$, Michael F. Berger ${ }^{3}{ }^{3}$, Nikolaus Schultz ${ }^{4}$, \\ Marc Ladanyi ${ }^{3}$, Richard J. O'Reilly', David H. Abramson' ${ }^{6}$, Ozge Ceyhan-Birsoy ${ }^{3}$, Liying Zhang ${ }^{3}$, \\ Diana Mandelker ${ }^{3}$, Neerav N. Shukla', Andrew L. Kung ${ }^{1}$, Kenneth Offit ${ }^{2}$, Ahmet Zehir ${ }^{3 凶}$ and \\ Michael F. Walsh ${ }^{1,2} \bowtie$
}

The spectrum of germline predisposition in pediatric cancer continues to be realized. Here we report 751 patients with solid tumors who underwent prospective matched tumor-normal DNA sequencing with downstream clinical use (ClinicalTrials.gov NCT01775072). Germline pathogenic and likely pathogenic (P/LP) variants were reported. One or more P/LP variants were found in 18\% (138/751) of individuals when including variants in low-, moderate- and high-penetrance dominant or recessive genes or in 13\% (99/751) of individuals in moderate- and high-penetrance dominant genes. Thirty-four percent of high- or moderate-penetrance variants were unexpected based on the patient's diagnosis and previous history. Seventy-six percent of patients with positive results completed a clinical genetics visit, and $21 \%$ had at least one relative undergo cascade testing as a result of this testing. Clinical actionability additionally included screening, risk reduction in relatives, reproductive use and targeted therapies. Germline testing should be considered for all children with cancer.

$\mathrm{P}$ ediatric cancer is rare, with fewer than 10,000 solid tumors diagnosed in children annually in the United States ${ }^{1}$. Previous studies interrogating germline predisposition broadly across pediatric cancer types have found heritable germline predisposition in $8-12 \%$ of patients. The yield of germline predisposition detected is dependent on the genes included for analysis and variant interpretation as well as the ascertainment biases found in each cohort. Iterative data are required to expand upon the understanding of susceptibility to pediatric cancer and determine the extent to which germline data may translate into clinical practice $e^{2-7}$.

Certain pediatric cancer diagnoses have well-established associations with germline mutations in specific genes and should automatically prompt clinical suspicion of a cancer predisposition, for example, retinoblastoma (RB1), pleuropulmonary blastoma (DICER1), optic pathway glioma (NF1), atypical teratoid/rhabdoid tumors (SMARCB1), small cell hypercalcemic ovarian tumors (SMARCA4), adrenal cortical tumors (TP53) and hypodiploid acute lymphoblastic leukemia (TP53) ${ }^{8-10}$. Germline testing can also be critical for distinguishing between conditions like neurofibromatosis type 1 (NF1) and constitutional mismatch repair deficiency (CMMRD), which can be phenocopies of each other. For example, a child presenting with numerous café au lait spots and leukemia may have either of these conditions, but treatment and screening recommendations for the proband and family members will differ depending on the germline diagnosis ${ }^{11}$.

Besides the known associations of causal germline mutations, broad tumor-normal sequencing has revealed novel associations ${ }^{9,12}$. While some of these findings likely represent population detection and do not play a role in the pathogenesis of the cancer in question ${ }^{13}$, other novel associations are likely causal. Population detection

'Department of Pediatrics, Memorial Sloan Kettering Cancer Center, New York, NY, USA. ${ }^{2}$ Department of Medicine, Memorial Sloan Kettering Cancer Center, New York, NY, USA. ${ }^{3}$ Department of Pathology, Memorial Sloan Kettering Cancer Center, New York, NY, USA. ${ }^{4}$ Department of Epidemiology and Biostatistics, Memorial Sloan Kettering Cancer Center, New York, NY, USA. ${ }^{5}$ Sloan Kettering Institute, Memorial Sloan Kettering Cancer Center, New York, NY, USA. ${ }^{6}$ Department of Surgery, Memorial Sloan Kettering Cancer Center, New York, NY, USA. ${ }^{7}$ These authors contributed equally:

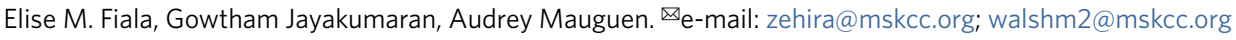


Table 1 | Broad germline sequencing studies of pediatric cancer with $>100$ patients

\begin{tabular}{|c|c|c|c|c|}
\hline Study & Hematologic & CNS & Solid tumor (non-CNS) & $\begin{array}{l}\text { Percent reported with germline } \mathrm{P} / \mathrm{LP} \text { variants in high/ } \\
\text { moderate-penetrance cancer predisposition genes }\end{array}$ \\
\hline Michigan: Mody et al. ${ }^{3}$ & 30 & 8 & 64 & 10 \\
\hline St. Jude: Zhang et al. ${ }^{2}$ & 588 & 245 & 287 & 8 \\
\hline Baylor: Parsons et al. ${ }^{6}$ & & 56 & 94 & 10 \\
\hline Columbia: Oberg et al. ${ }^{5}$ & 36 & 16 & 49 & 10 \\
\hline Australia: Wong et al. ${ }^{7}$ & 43 & 92 & 112 & 11 \\
\hline
\end{tabular}

Table 2 | Patient characteristics

\begin{tabular}{|c|c|c|c|c|}
\hline Characteristic & Mutation carriers, $n=138^{\mathrm{a}}$ & Non-mutation carriers, $n=613^{a}$ & Overall, $n=751$ & $P$ value $^{b}$ \\
\hline Sex & & & & 0.65 \\
\hline Female & $67(49 \%)$ & $282(46 \%)$ & $349(46 \%)$ & \\
\hline Male & $71(51 \%)$ & $331(54 \%)$ & $402(54 \%)$ & \\
\hline Age at diagnosis (years) & $7.4 \pm 6.8(0.0-19.0)$ & $8.4 \pm 6.3(0.0-19.0)$ & $8.2 \pm 6.4(0.0-19.0)$ & 0.11 \\
\hline Multiple diagnoses & & & & $1.519 \times 10^{-4}$ \\
\hline No & $124(90 \%)$ & $595(97 \%)$ & $719(96 \%)$ & \\
\hline \multicolumn{5}{|l|}{ Deceased } \\
\hline No & $117(85 \%)$ & $519(85 \%)$ & $636(85 \%)$ & \\
\hline Yes & $21(15 \%)$ & $94(15 \%)$ & $115(15 \%)$ & \\
\hline
\end{tabular}

aStatistics are presented as $n(\%)$ or mean \pm s.d. (minimum-maximum). bStatistical tests performed included chi-square tests of independence and $t$-tests, all two sided.

is expected, especially in large studies given the frequency of common germline predisposition; BRCA1/BRCA2 mutations are found in $2-3 \%$ ( 1 in 40 ) of individuals of Ashkenazi Jewish ancestry, and Lynch syndrome is detected in approximately $0.3 \%$ ( 1 in 300$)$ of the general population ${ }^{14}$. Even in the case of detecting apparently unrelated germline mutations, this information can be useful for adult family members through cascade testing and initiation of recommended screening and prevention measures for relatives who may not otherwise have come to medical attention ${ }^{15,16}$. In cases where functional assessment and/or enrichment analysis has supported causality, these findings have resulted in an expansion of the cancer spectrum of known syndromes (that is, $S D H x$ mutations with neuroblastoma and BRCA2 and PALB2 mutations with medulloblastoma $)^{9,12}$. However, for many observed associations, functional assessment and/or enrichment analysis has not yet been done, and it remains unknown what role, if any, the germline mutation played in tumorigenesis.

Therapeutic options have expanded for certain adult patients with cancer who have BRCA1, BRCA2, MLH1, MSH2, MSH6 and $P M S 2$ germline mutation-driven tumors ${ }^{17,18}$. In pediatric oncology, the main successes of germline-targeted therapies so far have been CMMRD-related tumors responding to immunotherapy, NF1-associated inoperable plexiform neurofibromas responding to selumetinib and NF1-associated optic pathway gliomas responding to MEK inhibitors ${ }^{19-21}$. The efficacy of other targeted therapies, for example, against BRCA1- and BRCA2-associated pediatric tumors (that is, subsets of medulloblastoma), remains preclinical and theoretical at this time ${ }^{22}$.

We have previously reported that the MSK-IMPACT custom-matched tumor-normal sequencing platform can identify a wide spectrum of germline mutations in adult patients with cancer and reveal new genetic associations ${ }^{23}$. Here we report findings from our pediatric population who underwent testing via the
MSK-IMPACT platform, including the prevalence and spectrum of $\mathrm{P} / \mathrm{LP}$ germline variants in cancer predisposition genes, associations between germline status and somatic profile, and subsequent clinical use of this information.

\section{Results}

Patient characteristics. During the study period, 751 patients had tumor-normal testing with consent for return of germline results. This represents an addition of $44 \%$ to the total number of cases of germline data reported from large sequencing studies of pediatric cancer (Table 1). Clinical characteristics of the patients are listed in Table 2. Patients were relatively evenly distributed between males and females (54\% versus $46 \%$ ) and had a mean age at diagnosis of 8.2 years. Age at diagnosis and sex were not significantly associated with the likelihood of detecting a P/LP variant.

The cancer distribution in our cohort included sarcoma $(30 \%$; $n=229)$, neuroblastoma (24\%; $n=182$ ), central nervous system (CNS) tumors $(19 \% ; n=143)$, retinoblastoma $(9 \% ; n=70)$ and other rare solid tumors $(19 \% ; n=143)$. Thirty-two individuals $(4 \%)$ had multiple tumors; hence, the percentages total $>100 \%$. In addition to a solid tumor, seven patients in our cohort had leukemia that developed either before the solid tumor from which they were in remission or after IMPACT was sent for their solid tumor. In comparison with the International Childhood Cancer Consortium distribution of solid tumors, our cohort is widely representative of all major groups, with enrichment of neuroblastoma, retinoblastoma and osteosarcoma and relatively few germ cell tumors, CNS tumors, thyroid cancers and melanomas, which reflects the patient population seen at our hospital (Fig. 1a).

Results reporting. Our process for analyzing and returning germline results involved expertise from Diagnostic Molecular Pathology and the Clinical Genetics Service, with input from a 
a

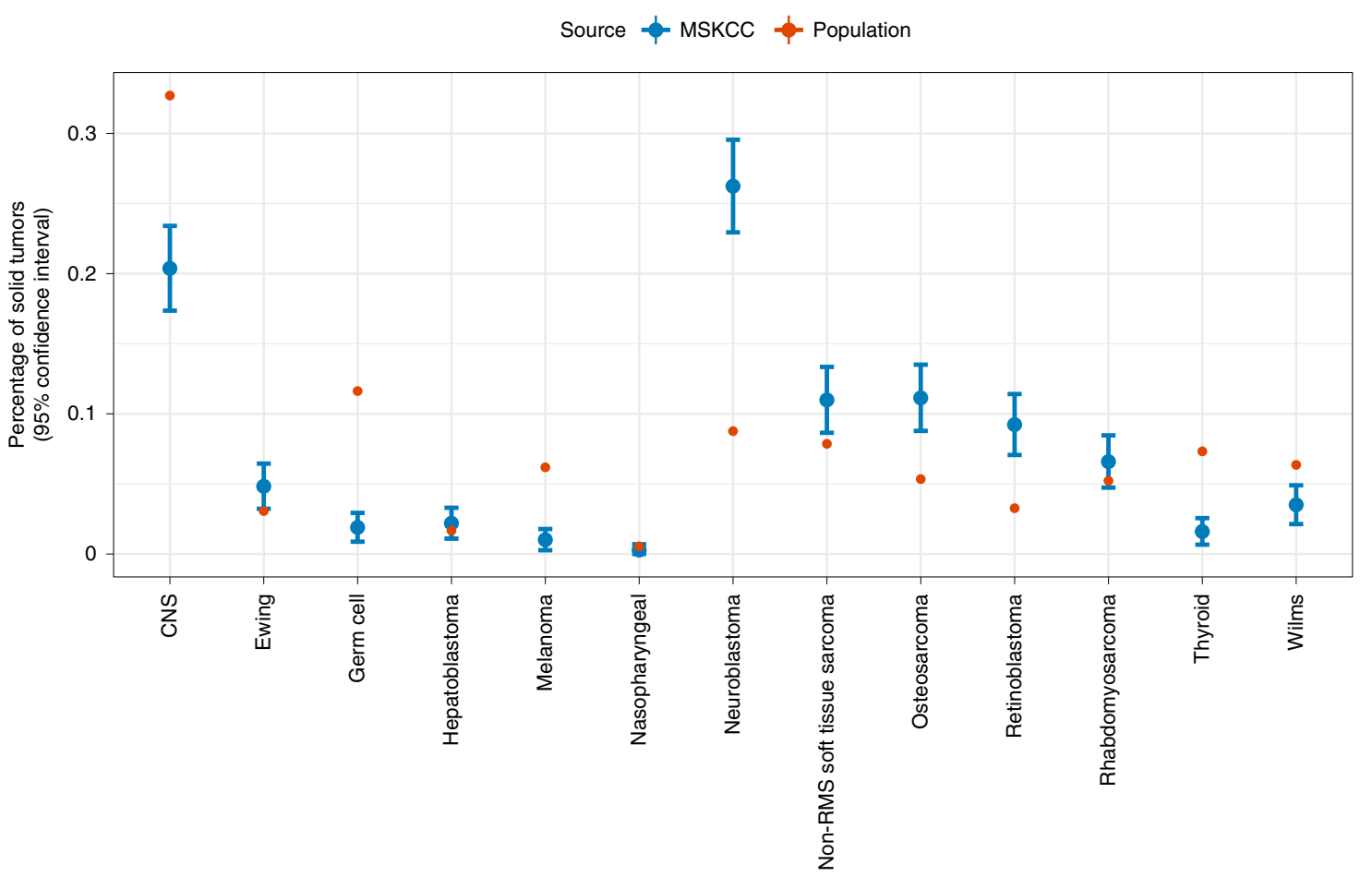

Diagnosis

b

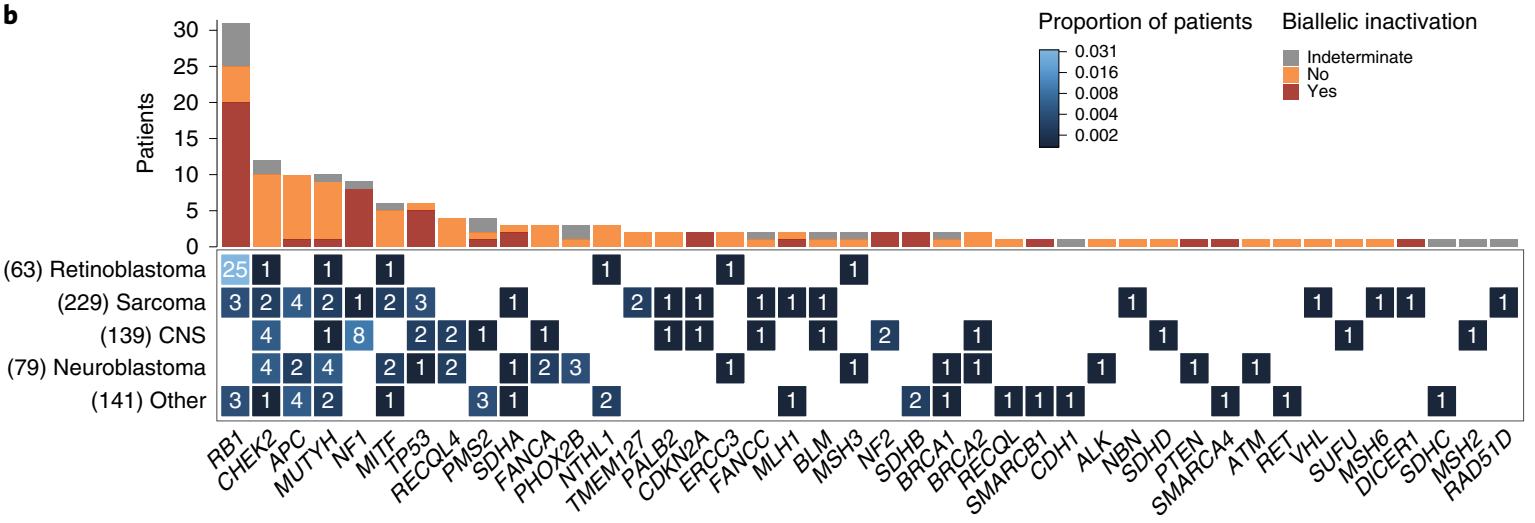

Fig. 1 | Cancer distribution and germline cancer predisposing mutations detected in MSKCC pediatric patient cohort. a, Cancer types represented in the current study (blue) compared to the Annual International Childhood Cancer Classification (AICCC) distribution (red). Current study data are based on $n=751$ patients and are shown with 95\% confidence intervals. US Census data (https://www.census.gov/quickfacts/fact/table/US/PST0452190) were used with AICCC data to calculate population percentages. RMS, rhabdomyosarcoma; MSKCC, Memorial Sloan Kettering Cancer Center. b, Germline $\mathrm{P} / \mathrm{LP}$ variants and biallelic inactivation across 138 patients in the cohort. Bottom, the heat map shows the occurrence of germline P/LP variants across genes and cancer types. Color indicates the proportion of patients with a particular cancer type and germline alteration, and the number on the tiles indicates the absolute count. The total number of patients in the cohort for each of the cancer types is displayed on the left. Top, the absolute count of patients with biallelic inactivation in the genes is shown on the $x$ axis. Biallelic inactivation includes $\mathrm{LOH}$ and second somatic hits. Patients for whom the tumor allele status could not be assessed due to insufficient tumor purity, unavailability of sufficient tumor tissue or low sequencing coverage in the tumor are indicated as indeterminate for biallelic inactivation.

multidisciplinary molecular tumor board when disease or treatment context was important. An example illustrating the value of this approach was in the assessment of the TP53 variant p.Arg196Leu. Based on the existing annotation in ClinVar, this variant was previously classified as a variant of unknown significance (VUS). However, taking into account hotspot data from the cBioPortal database and tumor histology (choroid plexus carcinoma), we used the ClinGen framework for integrating somatic tumor data for germline variant interpretation to consider this variant as LP and returned this finding to the family ${ }^{24}$.
Frequency and spectrum of germline variants. A total of 138 patients were positive for one or more P/LP variants (18\%) when including low-, moderate- and high-penetrance variants in recessive and dominant genes, and 13\% (99/751) were positive when including moderate- and high-penetrance mutations in dominant genes. P/LP variants were reported in $49 \%(34 / 70)$ of patients with retinoblastoma, $21 \%(30 / 143)$ of patients with CNS tumors, $15 \%$ $(28 / 182)$ of patients with neuroblastoma, $12 \%(28 / 229)$ of patients with sarcoma and $19 \%(27 / 143)$ of patients with other tumor types. The spectrum of variants found in patients with specific disease 
Table 3 | Percentage of positive cases by selected tumor types

\begin{tabular}{|c|c|c|c|}
\hline Tumor type & Total cases & Positive cases (\%) & Genes with $\mathrm{P} / \mathrm{LP}$ variants \\
\hline Astrocytoma & 27 & $5(19 \%)$ & BRCA2, SDHD, FANCC, CHEK2, RECQL4 \\
\hline Desmoplastic small round cell tumor & 16 & $1(6 \%)$ & PALB2 \\
\hline Ependymoma & 14 & $3(21 \%)$ & $N F 1, N F 2, F A N C A$ \\
\hline Ewing sarcoma & 31 & $5(16 \%)$ & MLH1, CHEK2, TMEM127, MITF, APC I1307K (2) \\
\hline Ganglioglioma & 7 & $1(14 \%)$ & SUFU \\
\hline Germ cell tumor & 17 & $2(12 \%)$ & PMS2, APC 11307K \\
\hline Glioblastoma & 13 & $3(23 \%)$ & PMS2 (homozygous), NF1, CHEK2, MUTYH \\
\hline Hepatoblastoma & 4 & $1(25 \%)$ & PMS2 \\
\hline Hepatocellular carcinoma & 11 & $1(9 \%)$ & MUTYH \\
\hline Malignant peripheral nerve sheath tumor & 7 & $7(100 \%)$ & NF1 (6), MSH2 \\
\hline Osteosarcoma & 74 & $7(9 \%)$ & RB1 (3), TP53, RAD51D, CDKN2A, MUTYH \\
\hline Retinoblastoma & 70 & $34(49 \%)$ & RB1 (31), ERCC3, MITF, CHEK2, MUTYH (2), MSH3, NTHL1 \\
\hline Rhabdomyosarcoma & 45 & $9(20 \%)$ & DICER1, TP53, MSH6, VHL, NBN, APC I1307K (2), FANCC \\
\hline Wilms tumor & 25 & $2(8 \%)$ & APC I1307K, MUTYH \\
\hline
\end{tabular}

Genes in which P/LP variants were detected are listed by disease type. Note that some patients had multiple P/LP variants; hence, some tumor types have more genes with P/LP variants listed than positive cases. See Supplementary Tables 1-4 for further information.

types is shown in Table 3. All variants were heterozygous with the exception of one individual with a homozygous PMS2 LP variant and two RB1 $\mathrm{P}$ variants that were mosaic. A full description of all patients and variants is in Supplementary Table 1.

The most common P/LPvariants in high-or moderate-penetrance genes were in RB1 $(n=31 ; 4 \%), N F 1(n=9 ; 1 \%)$ and TP53 $(n=6$; $1 \%)$ in patients with retinoblastoma and previous clinical diagnoses of NF1 or Li Fraumeni syndrome (LFS)-associated tumors, respectively (Table 3). Moderate- or high-penetrance P/LP variants were also detected in DNA damage repair genes $(n=24,3.2 \%$ of patients; $A T M, n=1 ; B R C A 1, n=2 ; B R C A 2, n=2 ; C D K N 2 A, n=2 ; C H E K 2$, $n=6$; ERCC3, $n=2 ; M L H 1, n=2 ; M S H 2, n=1 ; M S H 6, n=1 ;$ PMS2, $n=4$; RAD51D, $n=1)$, the RAS-MEK or mTOR-PTEN pathway ( $n=12,1.6 \%$ of patients; NF1, $n=9 ; N F 2, n=2 ;$ PTEN, $n=1)$ and metabolic pathways related to cancer $(n=8,1.1 \%$ of patients; SDHA, $n=3$; SDHB, $n=2$; SDHC, $n=1$; SDHD, $n=1$; VHL, $n=1)$. Seven individuals $(0.9 \%)$ harbored a second P/LP variant in a different cancer predisposition gene. One individual was found to have two variants in FANCC. This patient's phenotype was not consistent with Fanconi anemia (the tumor type was a pilocytic astrocytoma with no extreme reaction to chemotherapy), but the patient did not complete a Clinical Genetics visit, so it was not determined whether these variants are in cis or trans.

Thirty-four percent (34/101) of high/moderate-penetrance variants were unexpected based on the patient's diagnosis and previous history, and 50\% (35/70) were unexpected when excluding RB1 P/LP variants (Supplementary Table 1 ). Individuals who tested positive for a P/LP variant were more likely than those who tested negative to have had multiple primary cancer diagnoses (10\% versus $3 \%, P<0.001$; Table 2 ). Of 14 mutation carriers with multiple diagnoses, 7 individuals (50\%) had multiple cancers associated with their germline mutation (hereditary retinoblastoma, NF1 and NF2), and 2 were known to have therapy-related cancer, including one child with LFS who developed a therapy-related leukemia. Family history of childhood cancer was reported in 13\% (14/105) of individuals in our cohort with P/LP variants who completed a Clinical Genetics visit.

\section{Correlation between germline genotype and tumor pheno-} type. We were able to evaluate loss of heterozygosity (LOH) status for $120 \mathrm{P} / \mathrm{LP}$ variants, including 80 high- or moderate-penetrance variants. Variants were considered associated with biallelic loss in the tumor if $\mathrm{LOH}$ or a second somatic hit was present or in the case of the germline homozygous PMS2 LP variant. Germline P/LP variants in moderate- or high-penetrance genes were associated with biallelic loss in 55\% of individuals (44/80) (Figs. 1b and $2 \mathrm{a}$ and Supplementary Table 1). Of 53 germline P/LP variants with somatic data available that were considered expected based on the patient's tumor type or previous history, 43 (81\%) had LOH, a second somatic variant or germline biallelic loss. In comparison, $27 \mathrm{high} /$ moderate-penetrance germline $\mathrm{P} / \mathrm{LP}$ variants with somatic data available were considered unexpected, and just 2 (7\%) of these were associated with $\mathrm{LOH}$ (both CDKN2A variants, one in a case of osteosarcoma and one in a low-grade neuroepithelial tumor).

Clinical genetics follow-up. All 138 patients with positive results were referred to the Clinical Genetics Service, and 105 (76\%) have completed an appointment as of paper acceptance (Fig. 2a). Reasons for declining an appointment, when given, included previous knowledge and counseling on the mutation identified, declining patient status/death or being overwhelmed by disease/treatment and wishing to defer discussion until completion of therapy. Appointments were conducted in person (or via telemedicine during COVID-19) 
for all autosomal dominant mutations and by patient/clinician preference or by phone for carrier status of autosomal recessive mutations. Additionally, 116 of 613 patients (19\%) with negative results completed a visit with the Clinical Genetics Service. Five patients (including two sisters) with negative IMPACT testing were diagnosed with a cancer predisposition from additional testing (Fig. 2a).

Utility of germline results. Cascade testing was completed as a result of IMPACT testing in at least one family member for 29 of 138 individuals (21\%) with a P/LP variant detected (Fig. 2a). This resulted in the detection of 27 mutation carriers who were not previously aware of their status. In 14 additional cases (10\%, all of which were high/moderate-penetrance genes), there was documentation in the chart that family member testing had previously taken place, either because the variant detected on IMPACT was previously detected with the initiation of cascade testing or because the family members had previously been referred for genetic testing due to their own personal and/or family history of cancer. It should be noted that this number only reflects cascade testing that was completed through our clinic or done at an outside institution with records provided. Many families were provided with contact information for local genetic counseling services and may have pursued testing elsewhere.

Likelihood to undergo cascade testing was associated with the penetrance of the mutation detected and varied by gene; 37 of 99 patients (37\%) with a high/moderate-penetrance variant had or had previously had at least one family member undergo cascade testing compared to 6 of 39 patients (15\%) who only had a low-penetrance or autosomal recessive variant $(P=0.01)$. All six patients with germline TP53 P/LP variants were found to have inherited the variants from a parent, half of which were detected following IMPACT testing and half of which were previously known to the family. Cascade testing was completed less often in the families of probands identified to have P/LP variants in RB1 (7 of 31 cases; $23 \%$ ) and NF1 ( 2 of 9 cases; $22 \%$ ). Six patients were found to have expected de novo mutations (confirmation of paternity was not performed) in $R B 1$ (two cases), PHOX2B, SMARCA4, NF1 and, surprisingly, MLH1.

Patients identified to have mutations predisposing them to cancer in their age range were referred to our surveillance clinic, or additional screening was discussed with the primary attending if the patient was actively being treated. Sixty-seven patients had mutations associated with risk of cancer in childhood and thus screening recommendations in childhood (although 36 of these were variants in $R B 1$ in individuals with bilateral retinoblastoma or variants in NF1 in individuals meeting clinical criteria, hence not changing recommendations $)^{25,26}$. Family members identified to carry P/LP variants through cascade testing were similarly referred. This resulted in the detection of multiple tumors. A patient identified to have a germline SDHD mutation was found to have a carotid body paraganglioma on screening magnetic resonance imaging (MRI). In a family identified to have a TP53 mutation following the proband's diagnosis of rhabdomyosarcoma, the father was subsequently diagnosed with thyroid carcinoma and meningioma on whole-body MRI (Fig. 2b). Additionally, in one family without a prior cancer predisposition diagnosis, identifying the germline TP53 mutation led to the birth of an unaffected child who was conceived via in vitro fertilization (IVF) and preimplantation genetic testing (PGT).

Seven patients $(0.9 \%)$ were identified in our cohort with a heterozygous $\mathrm{P} / \mathrm{LP}$ variant in a mismatch repair (MMR) gene ( $M L H 1$, MSH2, MSH6 and PMS2), which is significantly higher than the population frequency previously reported in the Healthy Nevada Project of $0.03 \%(P=0.0095)$ (Supplementary Table 4$)^{14}$. In addition, one patient with a glioblastoma had a homozygous LP variant in PMS2 diagnostic of CMMRD. The patient's tumor was microsatellite instability-high (MSI-H) and had a very high tumor mutational burden of 243 mutations per megabase, consistent with CMMRD-related disease. The patient was treated with pembrolizumab but unfortunately progressed while on this treatment and died. Microsatellite analysis was available for six of the seven patients with heterozygous mutations. One patient with colon adenocarcinoma had an MSI-H tumor, while the other five were microsatellite stable (MSS) (none of these five tumors were associated with Lynch syndrome). Four patients with heterozygous mutations in the MMR genes had advanced disease. Two of these patients with advanced disease were prescribed immunotherapy based on their germline finding. One patient with an immature teratoma that had developed into a mature teratoma with glioma and spindle cell sarcoma and a PMS2 mutation exhibited a partial response to immunotherapy (Fig. 2c). Another patient with a de novo $M L H 1$ mutation and Ewing sarcoma died of disease.

\section{Discussion}

Cancer predisposition is found in high- or moderate-penetrance genes in $8-13 \%$ of patients with pediatric cancer according to this study and multiple previous pan-pediatric cancer studies ${ }^{2,3,5,6}$. As with previous studies, our study has an ascertainment bias based on the diagnoses of patients presenting to our center (Fig. 1a), and these numbers do not represent a truly unselected pediatric cancer cohort. The clinical utility of detecting a germline predisposition will depend on the individual patient and variant found but has not previously been studied in a systematic way across pediatric cancer. Our cohort underwent prospective Clinical Laboratory Improvement Amendments (CLIA)-validated in-house testing, enabling clinical actionability through our multidisciplinary molecular tumor board, Clinical Genetics Service and Pediatric Surveillance Clinic.

Even in the context of large pan-cancer next-generation sequencing (NGS) panels, it is important to recognize the limitations of the assay and to recognize which patients warrant additional testing. Many patients with negative results on MSK-IMPACT were seen by Clinical Genetics for this reason; in fact, more patients with negative results (116) were seen than patients with positive results (105). NGS-based assays are not designed to detect imprinting disorders or disorders of telomere biology in patients without a detectable mutation in a gene included on the panel ${ }^{8}$. Four patients in our cohort with negative IMPACT results had epimutations associated with Beckwith-Wiedemann syndrome, three of whom did not have prior diagnoses of this condition (as previously reported) ${ }^{27}$. Certain genetic changes will also be missed by panel-based testing, such as balanced translocations, some copy number alterations and repeat

Fig. 2 | Clinical translation resulting from detection of cancer predisposing mutations in MSKCC pediatric patient cohort. a, Universal germline sequencing in 751 patients undergoing tumor-normal sequencing using MSK-IMPACT, with tumor status of the alternate allele if available, screening recommendations when indicated, cascade testing in relatives and examples of clinical translation. One family reported undergoing IVF and PGT, which resulted in a pregnancy and birth of a child unaffected by LFS. While counseling using germline results in this manner was carried out by the Clinical Genetics team, the number of families using this information for reproductive purposes was not systematically followed. CGS, Clinical Genetics Service. b,c, Examples of clinical use of germline results. b. A proband with embryonal rhabdomyosarcoma was detected to have a germline TP53 mutation, which was subsequently found in the patient's father through cascade testing. The father was found through screening to have thyroid cancer and a meningioma. c, A proband with a teratoma was found to have a germline PMS2 mutation and was started on immunotherapy. Cascade testing has not been performed in this family. AACR, American Academy of Cancer Research. 
expansions (such as in $P H O X 2 B$ ). Genes with known pathogenic mutations in non-exonic regions (promoter or deep intronic mutations) need to have these regions specifically targeted to detect the mutations. For example, one patient in our cohort with a clinical diagnosis of NF1 but whose IMPACT testing only showed a germline CHEK2 mutation underwent additional clinical testing of $N F 1$,

a

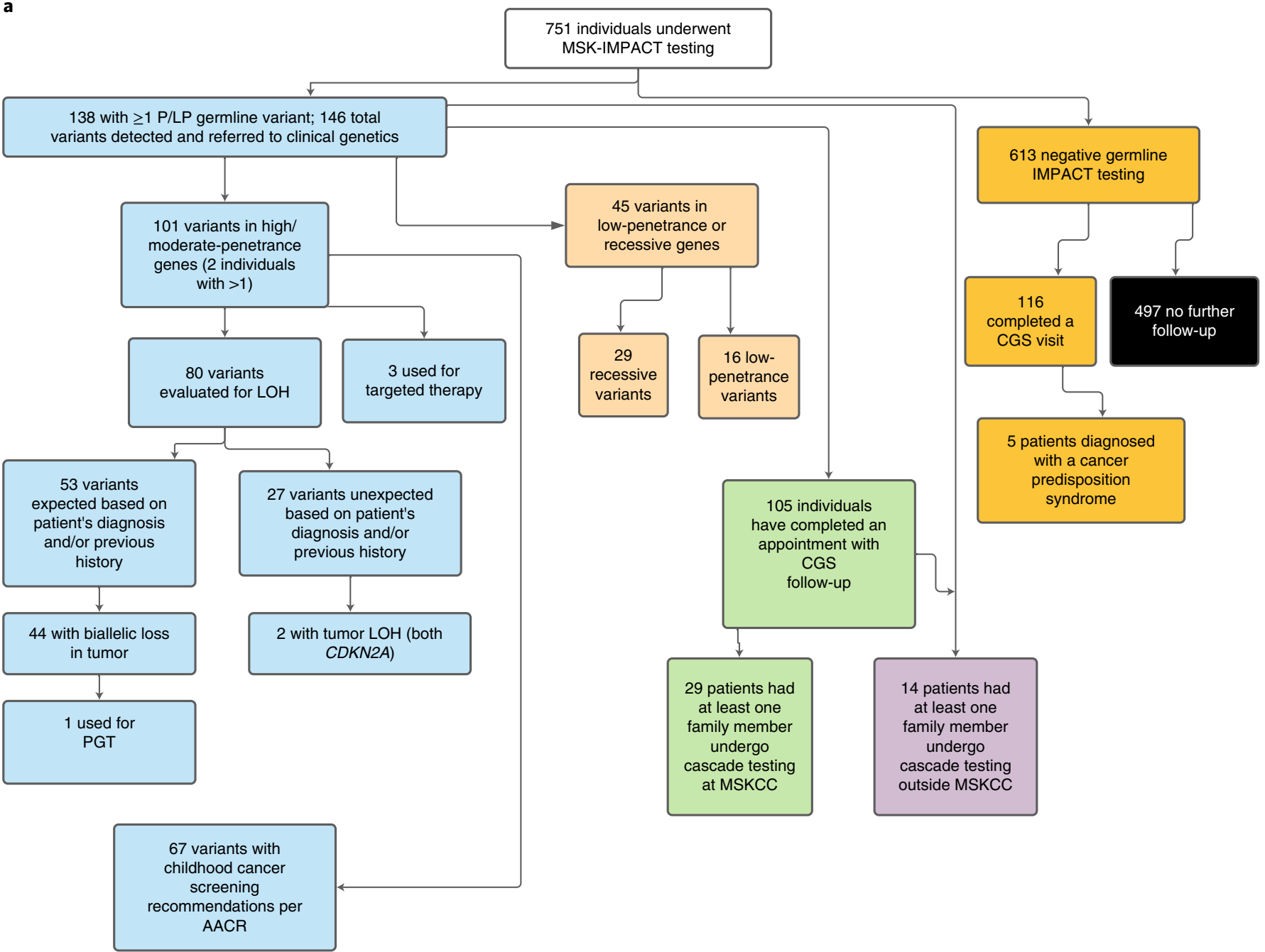

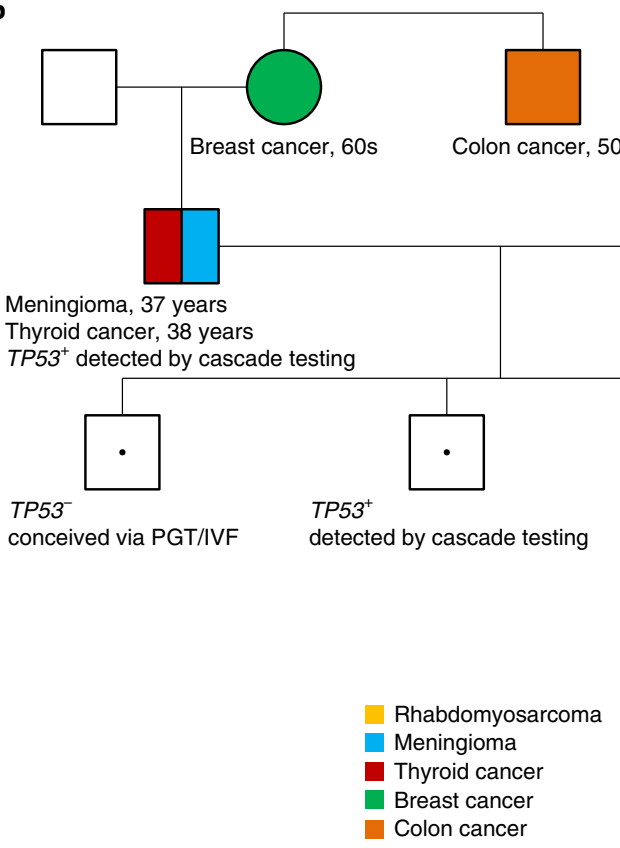

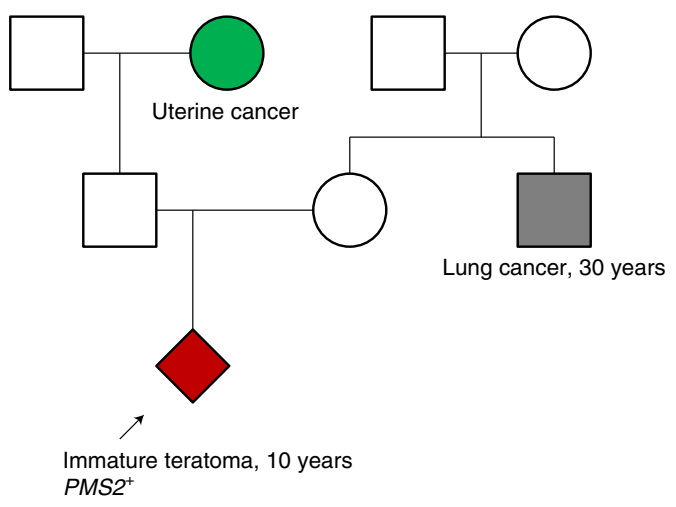

TP53 $^{+}$
Rhabdomyosarcoma, 4 years

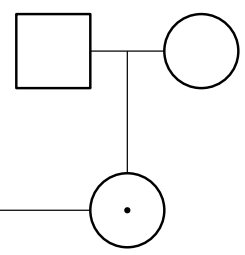

underwent cascade testing

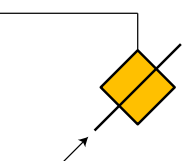

Immature teratoma

Uterine cancer

Lung cancer 
which revealed the deep intronic splicing $P$ variant c.5749+332A $>\mathrm{G}$. In some cases, lower-level mosaicism may be detected by a clinical test developed to have particularly high sequencing depth of one particular gene. Two patients (twin sisters) with juvenile xanthogranulomas in our cohort with negative IMPACT testing were found to be mosaic for an NF1 P variant that was below the level of detection of IMPACT. Knowledge of clinically relevant limitations for an individual patient is critical to guide appropriate follow-up testing, when needed, to maximize the detection of predisposition.

Some limitations of NGS panels may be addressed by incorporating additional technologies into the clinical workflow. RNA-based testing has recently been shown to detect $\mathrm{P}$ variants that would be missed altogether by DNA sequencing and to help clarify the pathogenicity of VUS in a substantial portion of cases $^{28,29}$.

Recommendations for germline testing of pediatric patients with cancer are often tumor specific or may take additional factors such as family history into account (that is, Chompret criteria for LFS ${ }^{30}$. However, consistent with previous studies ${ }^{23}$, we found a substantial number of patients with high/moderate-penetrance germline P/LP variants in unexpected genes given their cancer type or previous history. The role of these mutations in tumorigenesis is often not known, but some evidence for or against causality may be inferred from the patient's accompanying tumor data (that is, MSI status, second somatic hit, $\mathrm{LOH}$, tumor signature and so on) or from further study (that is, functional studies or enrichment studies). Our finding of two unexpected CDKN2A variants associated with tumor $\mathrm{LOH}$ is interesting and warrants further study to determine whether these rare tumor types represent an expansion of the phenotype, especially in the case of osteosarcoma as an enrichment of $C D K N 2 A$ germline mutations in this disease type has previously been reported ${ }^{31}$. These results underscore the importance of assessing tumor LOH, which may help point toward novel causal associations in the case of unexpected germline findings.

On a broader scale, enrichment data may suggest a causal relationship, such as our finding of more Lynch syndrome (heterozygous MMR P/LP variants) than would be expected at a population frequency. However, much further study of this potential association in additional cohorts is warranted, especially given that the tumors here were MSS, with the exception of colorectal cancer. More robust data on the response of such patients to immunotherapy may also guide understanding of the significance of these unexpected findings in pediatric cancers.

Targeted therapy informed by germline data has resulted in rare clinical responses in pediatric cancer (for example, CMMRD). However, the clinical utility of germline data informing therapy requires much further exploration. Data from studies in adults of targeted therapies for germline mutations have shown somewhat improved clinical outcomes in a larger number of cases ${ }^{18}$. It is very likely that the list of germline mutations associated with clinical trial eligibility and approved therapies will continue to grow. In addition to targeted therapies, germline data may also be useful in predicting which patients will experience increased therapeutic toxicity and may benefit from reduced dosing. In the past, this has been mainly considered in patients with rare bone marrow failure syndromes (that is, Fanconi anemia and dyskeratosis congenita); recently, however, certain heterozygous germline mutations in DNA repair genes (including in genes only associated with autosomal recessive disease) have been shown to be associated with increased risk of secondary cancers following radiation therapy or alkylating chemotherapy $^{32}$. If this association continues to be supported in further studies, there could be increasing clinical relevance to the patients themselves when detecting carrier status for certain autosomal recessive genes in terms of dosing considerations.

Additionally, cascade testing in families can multiply the benefits of detecting a germline mutation. Surveillance, risk reduction and reproductive planning can become available to family members in addition to the proband ${ }^{15,16}$. Despite the high pretest probability of detecting a P/LP variant, the relatively low cost and the straightforward interpretation (without VUS), uptake of cascade testing in family members at risk of a cancer predisposition has typically been suboptimal. Previous studies in adult cancer predisposition have generally found that approximately half of at-risk first-degree relatives undergo cascade testing ${ }^{33}$, with more recent initiatives investigating practices to identify and reduce burdens on family members to increase uptake $e^{16,34-36}$. Uptake in our cohort was similarly low, although variable based on penetrance of the variant as well as the gene itself (that is, a low cascade testing rate for NF1 is unsurprising given that the diagnosis or absence of a diagnosis can typically be made clinically in relatives).

An important consideration of germline testing in pediatric cancer is cost-effectiveness, which has yet to be rigorously studied in this area. While the hope is that appropriate germline testing can be clinically utilized in a way that is overall cost-effective to the larger system, evaluating this will take much more long-term follow-up of patients than we have performed in this study. Potential cost savings exist through cancer screening and early detection, prevention, PGT and potentially more effective therapeutics; however, there are also significant costs associated with each of these in addition to the costs of sequencing and clinical genetics visits. Studies of cost-effectiveness in this area to date have focused on individual pieces of this process, for example, cancer screening in patients with LFS $^{37}$. The full analysis of the effects of pan-pediatric cancer germline sequencing will require long-term follow-up, as costs may be largely up front (that is, sequencing), while cost savings may not be realized for many years (that is, through early tumor detection associated with need for less treatment).

While disease- and family history-based testing guidelines have utility in detecting a portion of children with underlying predisposition, it is critical to recognize that a significant proportion of germline mutations will not be predicted based on these guidelines and will be missed if testing is restricted to only those meeting criteria. The potential for cancer predisposition should be considered for every child with cancer. Outside of guidelines-based testing, the potential additional utility of broader germline testing needs to be considered in the context of the patient's clinical condition (that is, potential to enroll in a clinical trial if certain mutations were detected) as well as the patient and family's desire for information that could primarily be used for other family members in the immediate future (that is, the finding of a BRCA1/BRCA2 mutation in a young child). Broad pan-cancer predisposition testing is a key part of research to appreciate the full spectrum associated with many germline mutations, but it also has increasing clinical utility for many patients.

\section{Methods}

Patient cohort. From July 2015 to July 2020, pediatric patients with solid tumors seen at Memorial Sloan Kettering Cancer Center (MSKCC) were offered matched tumor-normal DNA sequencing under an institutional protocol (ClinicalTrials. gov NCT01775072). Patients with sufficient tumor tissue available were offered enrollment at the physician's discretion and were shown a pretest video describing the testing or were seen by Clinical Genetics before having testing sent. Appropriate consents were obtained.

Patients were ascertained for this cohort based on being $\leq 19$ years old at diagnosis with the tumor that was used for sequencing. Clinical characteristics were collected from institutional electronic medical records. This study was approved by the MSKCC Institutional Review Board/Privacy Board.

Sequencing, variant interpretation and referral to clinical genetics. Tumor and blood samples were obtained and sequenced using the MSK-IMPACT platform, a capture-based NGS assay capable of identifying sequence mutations, copy number alterations and select gene fusions in 468 genes (341 and 410 genes in earlier versions), as described previously ${ }^{38,39}$. Germline data were analyzed in 88 genes (76 in the first version) (Supplementary Tables 2 and 3).

Variants were identified as described previously ${ }^{38,40}$. Germline variants were interpreted based on American College of Medical Genetics and Genomics (ACMG) guidelines by a clinical molecular geneticist or molecular pathologist ${ }^{41}$. When applicable, somatic data were utilized in accordance with ClinGen's 
framework for integrating somatic data for germline variant curation in cancer predisposition genes ${ }^{24}$. P/LP variants were reported while VUS were not.

Variants classified as VUS but with a high suspicion for pathogenicity were reviewed as part of a multidisciplinary molecular tumor board including molecular geneticists, molecular pathologists, clinical geneticists, oncologists and genetic counselors. In certain cases, after careful review of all molecular and clinical data, special interpretation was given to highly suspicious VUS, which were treated as $\mathrm{LP}$ and thus returned to these individuals with clinical recommendations.

Results were disclosed to the patient/parent by the ordering clinician. Patients with germline P/LP findings were referred for genetic counseling through the Clinical Genetics Service. Cascade testing was offered to family members of the proband as appropriate. Patients with negative results were referred to the Clinical Genetics Service at the discretion of the primary treating physician. Consultation was requested when there was suspicion of a predisposition condition that would not be detected via MSK-IMPACT (for example, Beckwith-Wiedemann syndrome), desire for clinical-grade testing of a particular gene or genes (to obtain VUS or known P/LP variants that would not be detected on MSK-IMPACT), concerning family history of cancer or based on the family's desire for genetic counseling. Additional clinical testing was sent if deemed appropriate by the Clinical Genetics Service and desired by the patient/family.

Statistics and reproducibility. No statistical method was used to predetermine sample size. No data were excluded from the analyses, and the experiment was not randomized.

According to known disease risks and prior modeling, $\mathrm{P} / \mathrm{LP}$ variants were classified at the gene level as high penetrance (relative risk (RR) of disease $>4$ ), moderate penetrance $(\mathrm{RR}, 2-4)$, low penetrance $(\mathrm{RR}<2)$ or were associated with autosomal recessive inheritance ${ }^{42}$. For $C H E K 2$ and $A P C$, penetrance classification was performed on the variant level; $A P C$ p.Ile1307Lys and CHEK2 p.Ile157Thr were considered low penetrance, and, if detected, other $A P C$ mutations were considered high penetrance and other CHEK2 mutations were considered moderate penetrance ${ }^{23,42}$

Somatic mutation data were also reported and considered to assess causality of identified germline variants. Estimates of tumor purity and somatic zygosity of all germline variants were evaluated using the FACETS algorithm (version 0.5.12) as described previously ${ }^{43,44}$. Tumor purity was estimated using mutant allele fractions or pathologist curation for cases where FACETS was unable to estimate purity. Biallelic inactivation was evaluated by inferring $\mathrm{LOH}$, defined as a loss of the wild-type allele in the tumor at the locus of a germline mutation. In the absence of $\mathrm{LOH}$ in a tumor, germline $\mathrm{P}$ variants were also considered biallelically inactivated if a second clonal or subclonal truncating mutation was observed in the same gene in the tumor or in the case of germline biallelic loss. Biallelic inactivation was considered indeterminate if (1) tumor tissue was not available for zygosity assessment, (2) tumor zygosity could not be evaluated because of germline $\mathrm{LOH}$ (3) tumor purity could neither be estimated by FACETS nor be estimated based on variant allele fraction or pathology curation, (4) read depth in the specimen at the locus of interest was less than 50 or (5) allele-specific copy number could not be evaluated by FACETS. MSI-H was defined as evidence of microsatellite instability at more than $10 \%$ of analyzed loci ${ }^{45}$.

$\mathrm{P} / \mathrm{LP}$ variants were considered expected or unexpected based on the patient's current diagnosis as well as any previous history (that is, history of previous tumor or of previous diagnosis of the germline predisposition detected). Family history was collected for patients who were seen by the Clinical Genetics Service.

The distribution of age at diagnosis, sex and presence of multiple diagnoses was compared between mutation carriers and non-mutation carriers using chi-square tests of independence and $t$-tests. Cascade testing rates were compared between genes with moderate/high penetrance and genes with low penetrance or autosomal recessive inheritance using Fisher's exact test. The frequency of Lynch syndrome detected in our cohort was compared to the population frequency previously reported in the Healthy Nevada Project ${ }^{14}$ using Fisher's exact test.

Reporting Summary. Further information on research design is available in the Nature Research Reporting Summary linked to this article.

\section{Data availability}

Deidentified clinical and molecular data for all patients reported in this study are available in Supplementary Table 1. P/LP variants reported here have been submitted to ClinVar with submission number SUB8689141.

Received: 5 June 2020; Accepted: 7 January 2021; Published online: 15 February 2021

\section{References}

1. Howlader, N. et al. (eds.) SEER Cancer Statistics Review, 1975-2014 (National Cancer Institute, 2017).

2. Zhang, J. et al. Germline mutations in predisposition genes in pediatric cancer. N. Engl. J. Med. 373, 2336-2346 (2015).

3. Mody, R. J. et al. Integrative clinical sequencing in the management of refractory or relapsed cancer in youth. JAMA 314, 913-925 (2015).
4. Harris, M. H. et al. Multicenter feasibility study of tumor molecular profiling to inform therapeutic decisions in advanced pediatric solid tumors: the individualized cancer therapy (iCat) study. JAMA Oncol. 2, 608-615 (2016).

5. Oberg, J. A. et al. Implementation of next generation sequencing into pediatric hematology-oncology practice: moving beyond actionable alterations. Genome Med. 8, 133 (2016).

6. Parsons, D. W. et al. Diagnostic yield of clinical tumor and germline whole-exome sequencing for children with solid tumors. JAMA Oncol. 2, 616-624 (2016)

7. Wong, M. et al. Whole genome, transcriptome and methylome profiling enhances actionable target discovery in high-risk pediatric cancer. Nat. Med. 26, 1742-1753 (2020)

8. Walsh, M. et al. in Abeloff's Clinical Oncology 6th edn (eds Niederhuber, J. E. et al.) Ch. 13 (Elsevier, 2020).

9. Holmfeldt, L. et al. The genomic landscape of hypodiploid acute lymphoblastic leukemia. Nat. Genet. 45, 242-252 (2013).

10. Scollon, S. et al. A comprehensive review of pediatric tumors and associated cancer predisposition syndromes. J. Genet. Couns. 26, 387-434 (2017).

11. Suerink, M. et al. Constitutional mismatch repair deficiency as a differential diagnosis of neurofibromatosis type 1: consensus guidelines for testing a child without malignancy. J. Med. Genet. 56, 53-62 (2019).

12. Gault, M. D. et al. Germline SDHA mutations in children and adults with cancer. Cold Spring Harb. Mol. Case Stud. 4, a002584 (2018).

13. Oak, N. et al. Ancestry-specific predisposing germline variants in cancer. Genome Med. 12, 51 (2020).

14. Grzymski, J. J. et al. Population genetic screening efficiently identifies carriers of autosomal dominant diseases. Nat. Med. 26, 1235-1239 (2020).

15. Walsh, M.F. et al. Germline BRCA2 mutations detected in pediatric sequencing studies impact parents' evaluation and care. Cold Spring Harb. Mol. Case Stud. 3, a001925 (2017).

16. Offit, K. et al. Cascading after peridiagnostic cancer genetic testing: an alternative to population-based screening. J. Clin. Oncol. 38, 1398-1408 (2020)

17. Le, D. T. et al. PD-1 blockade in tumors with mismatch-repair deficiency. N. Engl. J. Med. 372, 2509-2520 (2015).

18. Moore, K. et al. Maintenance olaparib in patients with newly diagnosed advanced ovarian cancer. N. Engl. J. Med. 379, 2495-2505 (2018).

19. Bouffet, E. et al. Immune checkpoint inhibition for hypermutant glioblastoma multiforme resulting from germline biallelic mismatch repair deficiency. J. Clin. Oncol. 34, 2206-2211 (2016).

20. Gross, A. M. et al. Selumetinib in children with inoperable plexiform neurofibromas. N. Engl. J. Med. 382, 1430-1442 (2020).

21. Fangusaro, J. et al. Selumetinib in paediatric patients with $B R A F$-aberrant or neurofibromatosis type 1-associated recurrent, refractory, or progressive low-grade glioma: a multicentre, phase 2 trial. Lancet Oncol. 20, 1011-1022 (2019)

22. van Vuurden, D. G. et al. PARP inhibition sensitizes childhood high grade glioma, medulloblastoma and ependymoma to radiation. Oncotarget 2, 984-996 (2011)

23. Mandelker, D. et al. Mutation detection in patients with advanced cancer by universal sequencing of cancer-related genes in tumor and normal DNA vs guideline-based germline testing. JAMA 318, 825-835 (2017).

24. Walsh, M. F. et al. Integrating somatic variant data and biomarkers for germline variant classification in cancer predisposition genes. Hum. Mutat. 39, 1542-1552 (2018)

25. Kratz, C. P. et al. Cancer screening recommendations for individuals with Li-Fraumeni syndrome. Clin. Cancer Res. 23, e38-e45 (2017).

26. Rednam, S. P. et al. Von Hippel-Lindau and hereditary pheochromocytoma/ paraganglioma syndromes: clinical features, genetics, and surveillance recommendations in childhood. Clin. Cancer Res. 23, e68-e75 (2017)

27. Fiala, E. M. et al. $11 \mathrm{p} 15.5$ epimutations in children with Wilms tumor and hepatoblastoma detected in peripheral blood. Cancer 126, 3114-3121 (2020).

28. Benayed, R. et al. High yield of RNA sequencing for targetable kinase fusions in lung adenocarcinomas with no mitogenic driver alteration detected by DNA sequencing and low tumor mutation burden. Clin. Cancer Res. 25, 4712-4722 (2019).

29. Karam, R. et al. Assessment of diagnostic outcomes of RNA genetic testing for hereditary cancer. JAMA Netw. Open 2, e1913900 (2019).

30. Knapke, S. et al. Hereditary cancer risk assessment in a pediatric oncology follow-up clinic. Pediatr. Blood Cancer 58, 85-89 (2012).

31. Mirabello, L. et al. Frequency of pathogenic germline variants in cancer-susceptibility genes in patients with osteosarcoma. JAMA Oncol. 6 724-734 (2020)

32. Qin, N. et al. Pathogenic germline mutations in DNA repair genes in combination with cancer treatment exposures and risk of subsequent neoplasms among long-term survivors of childhood cancer. J. Clin. Oncol. 38, 2728-2740 (2020)

33. Sharaf, R. N. et al. Uptake of genetic testing by relatives of Lynch syndrome probands: a systematic review. Clin. Gastroenterol. Hepatol. 11, 1093-1100 (2013) 
34. Courtney, E. et al. Impact of free cancer predisposition cascade genetic testing on uptake in Singapore. NPJ Genom. Med. 4, 22 (2019).

35. Frey, M. K. et al. Prospective feasibility trial of a novel strategy of facilitated cascade genetic testing using telephone counseling. J. Clin. Oncol. 38, 1389-1397 (2020).

36. Srinivasan, S. et al. Stakeholder perspectives on overcoming barriers to cascade testing in Lynch syndrome: a qualitative study. Cancer Prev. Res. 13, 1037-1046 (2020).

37. Tak, C. R. et al. Cost-effectiveness of early cancer surveillance for patients with Li-Fraumeni syndrome. Pediatr. Blood Cancer 66, e27629 (2019).

38. Cheng, D. T. et al. Memorial Sloan Kettering-integrated mutation profiling of actionable cancer targets (MSK-IMPACT): a hybridization capture-based next-generation sequencing clinical assay for solid tumor molecular oncology. J. Mol. Diagn. 17, 251-264 (2015).

39. Zehir, A. et al. Mutational landscape of metastatic cancer revealed from prospective clinical sequencing of 10,000 patients. Nat. Med. 23 , 703-713 (2017).

40. Cheng, D. T. et al. Comprehensive detection of germline variants by MSK-IMPACT, a clinical diagnostic platform for solid tumor molecular oncology and concurrent cancer predisposition testing. BMC Med. Genomics 10, 33 (2017).

41. Richards, S. et al. Standards and guidelines for the interpretation of sequence variants: a joint consensus recommendation of the American College of Medical Genetics and Genomics and the Association for Molecular Pathology. Genet. Med. 17, 405-424 (2015).

42. Tung, N. et al. Counselling framework for moderate-penetrance cancersusceptibility mutations. Nat. Rev. Clin. Oncol. 13, 581-588 (2016).

43. Shen, R. \& Seshan, V. E. FACETS: allele-specific copy number and clonal heterogeneity analysis tool for high-throughput DNA sequencing. Nucleic Acids Res. 44, e131 (2016).

44. Jonsson, P. et al. Tumour lineage shapes BRCA-mediated phenotypes. Nature 571, 576-579 (2019).

45. Niu, B. et al. MSIsensor: microsatellite instability detection using paired tumor-normal sequence data. Bioinformatics 30, 1015-1016 (2014).

\section{Acknowledgements}

This work was funded in part by the Robert and Kate Niehaus Center for Inherited Cancer Genomics, the Pediatric Translational Medicine Program, the Marie-Josée and Henry R. Kravis Center for Molecular Oncology and the National Cancer Institute Cancer Center Core grant number P30-CA008748.

\section{Author contributions}

M.F.W., E.M.F. and A.Z. conceptualized and designed the study. E.M.F., G.J., A. Mauguen, J.A.K., N.B., Y.K., M.H.F., A. Maio, E.E.S.-M., M. Sheehan, A.G.A., A.L., M.I.C., K.C., S. Murkherjee, E.K.S., T.T., J.G.B., P.A.M., L.W., F.S.D.C., N.-K.C., E.B., A.K., M.O., J.H.F., I.J.D., Y.K., S.G., S.F.S., C.J.F., M. Sulis, M.K., S. Modak, T.E.H., S.R.,
C.Y., S.J., J.V., S.T., D.N.F., Z.K.S., M.R., M.F.B., N.S., M.L., R.J.O.R., D.H.A., O.C.-B., L.Z D.M., N.N.S., A.L.K., K.O., A.Z. and M.F.W. acquired, analyzed and interpreted the data. M.F.W. and E.M.F. drafted the manuscript. G.J., A. Mauguen, J.A.K., N.B., Y.K., M.H.F., A. Maio, E.E.S.-M., M. Sheehan, A.G.A., A.L., M.I.C., K.C., S. Murkherjee, E.K.S., T.T., J.G.B., P.A.M., L.W., F.S.D.C., N.-K.C., E.B., A.K., M.O., J.H.F., I.J.D., Y.K., S.G., S.F.S., C.J.F., M. Sulis, M.K., S. Modak, J.T.G., T.E.H., S.R., C.Y., S.J., J.V., S.T., D.N.F., Z.K.S., M.R., M.F.B., N.S., M.L., R.J.O.R., D.H.A., O.C.-B., L.Z., D.M., N.N.S., A.L.K., K.O. and A.Z. critically revised the manuscript for important intellectual content. A. Mauguen performed the statistical analysis. M.F.W., A.L.K., K.O. and M.L. obtained funding E.M.F., J.A.K., N.B., Y.K., M.H.F., A. Maio, E.E.S.-M., M. Sheehan and A.G.A. performed administrative, technical or material support. M.F.W., A.Z., A.L.K. and K.O. supervised the study.

\section{Competing interests}

P.A.M.: Takeda, uncompensated speaking; Amgen, stock ownership; Ipsen, mock ODAC panel; Salarius, mock ODAC panel; P.A.M.'s spouse: Boehringer Ingelheim, consulting; Genentech, speakers' bureau; Eastern Pulmonary Society. N.K.C. holds ownership interest/equity in Y-mAbs Therapeutics, Inc., reports receiving commercial research grants from Y-mAbs Therapeutics, Inc., and Abpro Labs, Inc., holding ownership interest/equity in Abpro Labs and owning stock options in Eureka Therapeutics, Inc. N.K.C. is a scientific advisory board member of Abpro Labs and Eureka Therapeutics. N.K.C. is the inventor and owner of issued patents licensed by MSK to Y-mAbs Therapeutics, Biotec Pharmacon and Abpro Labs. I.J.D. has done consulting and advisory board activities with Apexigen, AstraZeneca, Bayer, Bristol-Myers Squibb/ Celgene, Fennec and Roche. I.J.D. has served as an institutional PI for pharma-sponsored clinical trials at MSKCC from Bristol-Myers Squibb, Genentech and Novartis. M.K. has consultant agreements with AstraZeneca, Bayer, CereXis, QED Therapeutics and Recursion Pharma (personal fees received). S. Modak. is a consultant to Y-mAbs Therapeutics, Inc., Illumina, Inc., and Progenics Pharmaceuticals. Z.K.S's immediate family member serves as a consultant for Allergan, Adverum Biotechnologies, Alimera Sciences, Fortress Biotech, Genentech/Roche, Novartis, Optos, Regeneron, Regenxbio and Spark Therapeutics.

\section{Additional information}

Supplementary information The online version contains supplementary material available at https://doi.org/10.1038/s43018-021-00172-1.

Correspondence and requests for materials should be addressed to A.Z. or M.F.W.

Peer review information Nature Cancer thanks Todd Druley, Heather Hampel and the other, anonymous, reviewer(s) for their contribution to the peer review of this work.

Reprints and permissions information is available at www.nature.com/reprints.

Publisher's note Springer Nature remains neutral with regard to jurisdictional claims in published maps and institutional affiliations.

(c) The Author(s), under exclusive licence to Springer Nature America, Inc. 2021 


\section{Reporting Summary}

Nature Research wishes to improve the reproducibility of the work that we publish. This form provides structure for consistency and transparency in reporting. For further information on Nature Research policies, see our Editorial Policies and the Editorial Policy Checklist.

\section{Statistics}

For all statistical analyses, confirm that the following items are present in the figure legend, table legend, main text, or Methods section.

$\mathrm{n} / \mathrm{a}$ Confirmed

$\bigotimes$ The exact sample size $(n)$ for each experimental group/condition, given as a discrete number and unit of measurement

Х $\square$ A statement on whether measurements were taken from distinct samples or whether the same sample was measured repeatedly

The statistical test(s) used AND whether they are one- or two-sided

Only common tests should be described solely by name; describe more complex techniques in the Methods section.

$\bigotimes \square$ A description of all covariates tested

Х $\square$ A description of any assumptions or corrections, such as tests of normality and adjustment for multiple comparisons

$\triangle$ A full description of the statistical parameters including central tendency (e.g. means) or other basic estimates (e.g. regression coefficient)

AND variation (e.g. standard deviation) or associated estimates of uncertainty (e.g. confidence intervals)

$\varnothing$ For null hypothesis testing, the test statistic (e.g. $F, t, r$ ) with confidence intervals, effect sizes, degrees of freedom and $P$ value noted Give $P$ values as exact values whenever suitable.

Х $\square$ For Bayesian analysis, information on the choice of priors and Markov chain Monte Carlo settings

Х $\square$ For hierarchical and complex designs, identification of the appropriate level for tests and full reporting of outcomes

Х $\square$ Estimates of effect sizes (e.g. Cohen's d, Pearson's $r$ ), indicating how they were calculated

Our web collection on statistics for biologists contains articles on many of the points above.

\section{Software and code}

Policy information about availability of computer code

$\begin{array}{ll}\text { Data collection } & \text { FACETS version } 0.5 .12 \\ \text { Data analysis } & \text { PRISM for statistical analysis and R version } 3.3 \text { (R Foundation) }\end{array}$

For manuscripts utilizing custom algorithms or software that are central to the research but not yet described in published literature, software must be made available to editors and

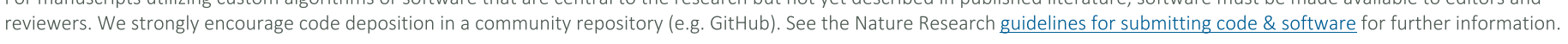

\section{Data}

Policy information about availability of data

All manuscripts must include a data availability statement. This statement should provide the following information, where applicable:

- Accession codes, unique identifiers, or web links for publicly available datasets

- A list of figures that have associated raw data

- A description of any restrictions on data availability

Deidentified clinical and molecular data for all patients reported in this study are available in the Supplementary Table. 
Please select the one below that is the best fit for your research. If you are not sure, read the appropriate sections before making your selection.

\ Life sciences

Behavioural \& social sciences

Ecological, evolutionary \& environmental sciences

For a reference copy of the document with all sections, see nature.com/documents/nr-reporting-summary-flat.pdf

\section{Life sciences study design}

All studies must disclose on these points even when the disclosure is negative. $\begin{array}{ll}\text { Sample size } & \text { All patients who were 0-19 years old at diagnosis and enrolled during the study period were included in the study sample, which resulted in } \\ n=751 \text {. This is the largest pediatric cohort of pan-solid tumor patients reported with germline findings from broad testing to date. }\end{array}$

Data exclusions All patients who consented and had testing performed were included. There were no exclusion criteria.

Replication Our methods include descriptions of the tumor-normal sequencing (including the gene lists in the supplementary table), that ACMG guidelines were used for variant classification, and clinical protocols for returning results to families and providing appropriate follow-up, and therefore should be reproducible by other cancer centers. All data of this nature in pediatric cancer will be biased based on the type of patients seen at a particular center, and for this reason a comparison of our cohort to the International Childhood Cancer Consortium Distribution of pediatric solid tumors and brain tumors was provided. We comment on comparisons to previous pediatric cancer germline sequencing studies, which found comparable levels of germline predisposition overall.

Randomization There was no randomization, all consenting patients had the same MSK-IMPACT test.

Blinding

Blinding was not relevant to our study as all patients who consented and enrolled had the same MSK-IMPACT test done.

\section{Reporting for specific materials, systems and methods}

We require information from authors about some types of materials, experimental systems and methods used in many studies. Here, indicate whether each material, system or method listed is relevant to your study. If you are not sure if a list item applies to your research, read the appropriate section before selecting a response.

\begin{tabular}{l|l}
\multicolumn{2}{l}{ Materials \& experimental systems } \\
\hline$n / a$ & Involved in the study \\
$\square$ & $\square$ Antibodies \\
$\square$ & $\square$ Eukaryotic cell lines \\
$\square$ & $\square$ Palaeontology and archaeology \\
$\square$ & $\square$ Animals and other organisms \\
$\square$ & $\bigotimes$ Human research participants \\
$\square$ & $\bigotimes$ Clinical data \\
$\square$ & $\square$ Dual use research of concern
\end{tabular}

\begin{tabular}{|c|c|}
\hline $\mathrm{n} / \mathrm{a}$ & Involved in the study \\
\hline X & ChIP-seq \\
\hline Х & Flow cytometry \\
\hline Х & MRI-based neuroimaging \\
\hline
\end{tabular}

\section{Human research participants}

Policy information about studies involving human research participants

Population characteristics diagnosis submitted for MSK-IMPACT testing, any other diagnoses, alive/deceased status, gender, age at diagnosis (for all diagnoses included)

Recruitment

Any attending within the Department of Pediatrics is able to consent patients to MSK-IMPACT. While all pediatric patients with solid tumors are eligible, there may be a bias towards patients suspected to have a predisposition being more likely to enroll, due to this being seen as more important by either the family and/or provider. Our study population also reflects the population of patients seen at MSKCC, which is compared in Flgure 1 to an international distribution.

Ethics oversight

This study was approved by the MSKCC Institutional Review Board/Privacy Board.

Note that full information on the approval of the study protocol must also be provided in the manuscript. 
Policy information about clinical studies

All manuscripts should comply with the ICMJE guidelines for publication of clinical research and a completed CONSORT checklist must be included with all submissions

Clinical trial registration

Study protocol

Data collection

Outcomes
ClinicalTrials.gov identifier: NCT01775072

https://clinicaltrials.gov/ct2/show/NCT01775072 and protocol available on request.

Patients were recruited within the MSK Department of Pediatrics between July 2015 and July 2020.

Patients with a pathogenic or likely pathogenic germline variant were considered to have positive test results and patients who did not have a germline pathogenic or likely pathogenic variant were given a negative test report. 